\title{
Syphilis Diagnosis and Treatment: State of The Art
}

\author{
Authors: \\ Emanuele Trovato, *Linda Tognetti, Marco Campoli, Elisa Cinotti, \\ Pietro Rubegni \\ Dermatology and Skin Bank Unit, Department of Clinical, Surgical, and Neurosciences, \\ University of Siena, Siena, Italy \\ *Correspondence to linda.tognetti@dbm.unisi.it \\ Acknowledgements: \\ Dr Trovato and Dr Tognetti contributed equally. \\ Received: \\ 01.09 .20 \\ Accepted: \\ 12.01.21 \\ Keywords: \\ Dermoscopy, diagnostic tests, syphilis, therapy. \\ Citation: \\ EMJ. 2021;DOI/10.33590/emj/20-00221.
}

\section{Abstract}

The present review summarises the current knowledge in the field of syphilis diagnosis and treatment, along with epidemiologic and historical data. A literature search was conducted in PubMed and Google Scholar, using the search terms "syphilis", "diagnosis", "dermoscopy", "management AND treatment", "laboratory tests AND syphilis", and "primary OR secondary OR tertiary OR congenital syphilis". A total of 55 out of 100 papers were included in this review. An overview of the different clinical presentation of primary, secondary, tertiary, and congenital syphilis, with particular attention to dermatologic signs and dermoscopic examination, is provided. The panorama diagnostic procedures are illustrated, along with their accuracy and recommendation. Treatment and management options of patients at different syphilis stages are provided and discussed according to the referring guidelines. The dermatologist can play a key role in providing the early and correct diagnosis and setting up in the proper management of patients with syphilis infection.

\section{BACKGROUND}

Syphilis is a chronic bacterial infection caused by Treponema pallidum subspecies pallidum, known by clinicians for hundreds of years and with more than 5 million new cases diagnosed every year worldwide, mostly in low- and middle-income countries., ${ }^{1,2}$ Syphilis can even be associated with other sexually transmitted diseases (STD), adverse pregnancy outcomes, and acceleration of HIV transmission.

The term 'syphilis' was first used in 1530 in a poem by Girolamo Fracastoro (in which Apollo curses the population with a disease that bears the name of the shepherd 'Syphilus', which angered him). Also called 'lues', plague, in Latin,

the disease is of importance to both individual and public health because it has a growing incidence. Although many consider this disease as specific to the 20th century, there has been a growing trend in recent years, especially among patients under the age of 25 . The fluctuating incidence of infection requires a greater effort for control and contagion management in order to avoid continuous transmission peaks. Syphilis incidence has again begun to increase dramatically in western Europe and the Americas, and now disproportionately occurs among males who have sex with males (MSM). ${ }^{3,4}$

T. pallidum subsp. pallidum is a slow-growing, motile spirochaete bacterium with a long spiral shape. Humans are its only natural host, and it cannot be cultured in vitro. It is closely related 
to other pathogenic treponemes, including $T$. pallidum subsp. pertenue, which causes yaws; Treponema carateum, which causes pinta; and T. pallidum subsp. endemicum, which causes endemic syphilis or bejel.

\section{EPIDEMIOLOGY}

The origins of syphilis remain to be controversial. ${ }^{5}$ According to the 'Columbian hypothesis', syphilis was brought to the Old World in 1493 by Columbus' returning seamen; on the other hand, the 'pre-Columbian hypothesis' states that the disease existed in both the Old and New Worlds prior to 1942; finally, the 'Evolutionary/Unitarian hypothesis' postulates that treponemal diseases were already distributed worldwide. $^{5}$ From the 1490s, the infection spread quickly across Europe, ${ }^{6}$ characterised by high virulence and mortality; however, in less than one century, less virulent strains were naturally selected.

From an epidemiological point of view, syphilis has been closely associated with HIV infection transmission. ${ }^{7-10}$ Syphilitic genital ulcers are densely infiltrated with lymphocytes (the primary target cells for HIV infection) and therefore provide a potential breach of entry for HIV acquisition, as well as a focus for HIV (and syphilis) transmission to others. ${ }^{11}$ Rates of primary and secondary syphilis among females have more than doubled between 2014 and 2018.12 The numbers of cases reported to the Centers for Disease Control and Prevention (CDC) increased by about 81\% from 2014 to 2018 in MSM. ${ }^{13}$ This happened especially in higher-income countries, where the use of geosocial network phone applications and/or location-based applications for sex led to an increased risk of sexually transmitted infections (STI), as well as to a reduced ability for partner notification because of anonymity. ${ }^{14,15}$ All stages of syphilis in pregnant females pose a risk of transmission to the fetus, but it is higher with early stages of infection than with later ones, so it is strictly important to perform an optimum and continuous prevention in these patients. ${ }^{16}$ Without treatment, the disease can progress over years through a series of clinical stages and may lead to irreversible neurological or cardiovascular complications.

\section{CLINICAL PRESENTATION}

\section{Primary Syphilis}

The primary stage of syphilis may be manifested clinically as a generally single, indurated, painless, and ulcerative chancre, which typically appears about 2-3 weeks after direct contact with another person's infectious lesion. ${ }^{3}$ Although chancres are most often seen on the penis, they can be located at nearly any place where direct contact with infectious lesions occurs, and sometimes it is unnoticed. ${ }^{17,18}$ The chancre begins as a macule, then papule, (Figure 1A) and rapidly develops into an erosion that is round or ovular in shape with sharp indurated margins (Figure 1B), which are pink, red, or greyish. The primary chancre can be accompanied by tender or nontender, painless, regional lymphadenopathy with overlying nonerythematous skin. Without treatment, after a period of 3-6 weeks, primary lesions spontaneously resolve without scarring. ${ }^{3}$ Sexual acquisition of syphilis occurs when an infectious lesion contacts the skin or mucous membrane of an uninfected person, often (but not exclusively) during oral, vaginal, or anal sex. The risk of transmission after sexual exposure is estimated at approximately 33\%. ${ }^{17}$

\section{Secondary Syphilis}

\section{Prodromic symptoms}

Secondary syphilis is the most recognised clinical syndrome of syphilis, mostly among females or MSM. Symptoms including malaise, myalgia, sore throat, headache, or low-grade fever commonly precede or are present during secondary syphilis.19,20 After 3-12 weeks from the resolution of a chancre or sometimes concurrently, secondary manifestations of infection result from haematogenous dissemination of spirochaetes.

\section{Syphilitic exanthema}

The rash of secondary syphilis is extraordinarily variable in appearance, involving both skin and mucous, and it could be localised or widespread. It generally appears as diffuse macular exanthema of 1-2 cm in diameter, on the trunk and extremities with scaly macules, or papules that are red-brown or 'ham coloured'. Lesions involving the palms of the hands or soles of the feet are diagnostic 

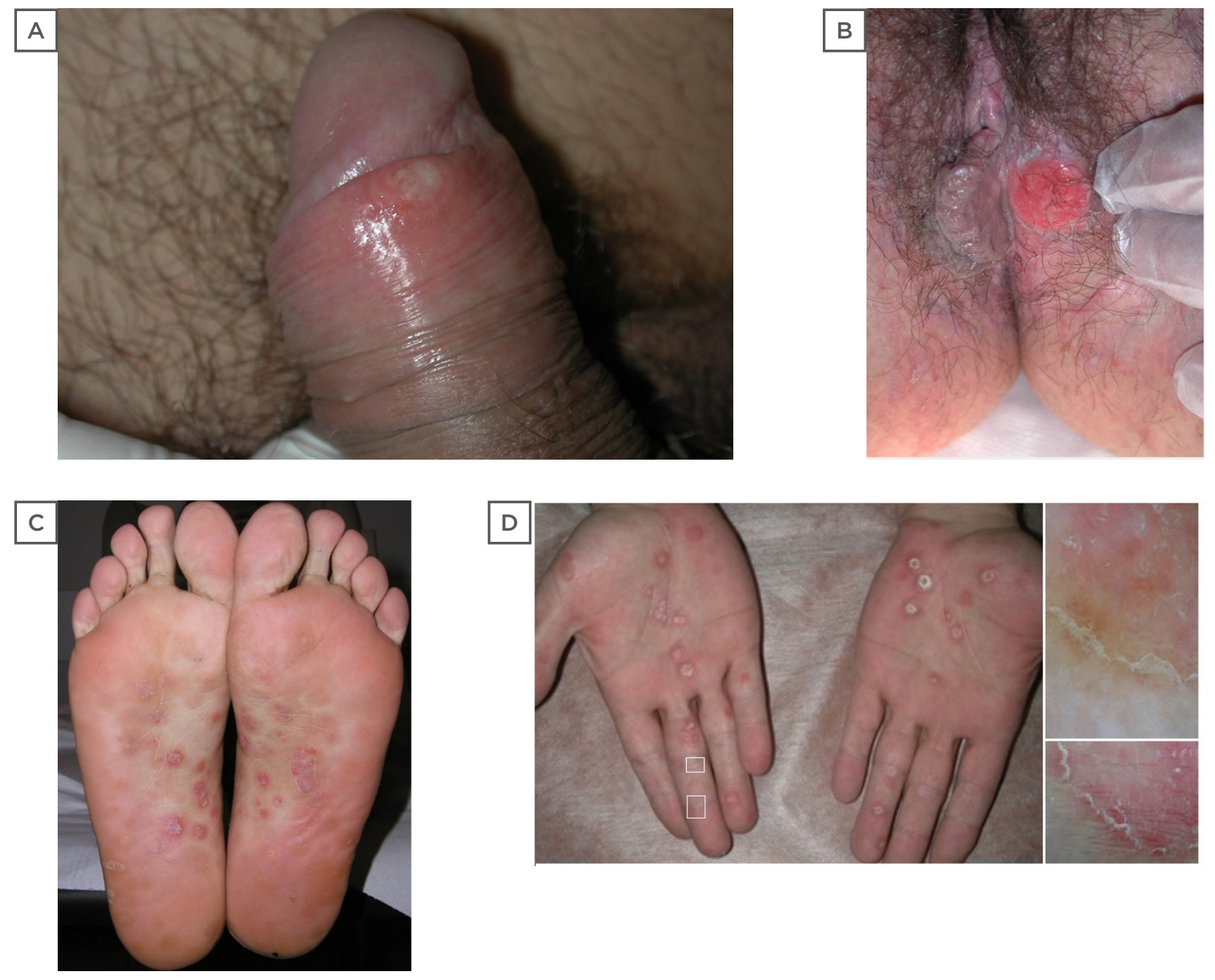

Figure 1: Clinical aspects of primary and secondary syphilis.

Two clinical presentations of the primary chancre, pathognomonic lesion of primary syphilis. A) Superficial erosion of the lesion at early stage on the glans in a 27-year-old male; B) advanced ulceration with raised indurated margins involving the perineal skin in a 34-year-old female. Secondary syphilis pathognomonic manifestations at palmoplantar sites consisting of erythematous papules with a central ring of scales (Biett's sign); C) soles of a 24-years-old male; D) and palms of a 45-year-old male. dermoscopic examination with contact polarised dermosocope (OM 17X) reveals an inward-oriented scaling collarette characterised by thick adherent white scales and erythermatous centre (D, squares).

Palmoplantar lesions are often pink, red, or brown macules or papules. Syphilis papules often show on their central surface a white ring of a scaling edge, known as Biett's collarette (Figure $1 C$ and D). In this phase, lesions can mimic any possible configuration, from annular to nodular, lichenoid, or psoriasis-like, hence the clinical differential diagnosis can be difficult. ${ }^{18-20}$

\section{Mucocutaneous and adnexal signs}

\section{Cutaneous manifestations are often} associated with diffuse lymphadenopathy, hepatosplenomegaly, hepatitis, alopecia, ${ }^{20}$ periostitis, or nephrotic syndrome. Even in secondary syphilis, lesions do not scar, and heal with postinflammatory hyperpigmentation. When mucous membranes are involved, lesions can appear as highly infectious mucous patches, sometimes with an exuberant, verrucous surface resembling a wart, called condyloma lata. ${ }^{19}$ Nail changes, including brittleness, splitting, pitting, onycholysis, onychomadesis, transverse grooves, and Beau lines, can occur. 


\section{Dermoscopic findings}

The morphology of this Biett's collarette can be highlighted by polarised dermoscopy, consisting of a circular scaling edge outward-directed with moderately adherent thick white scales. Moreover, the area inside the ring results de-epithelised, thus monomorphic dotted and glomerular vessels can be seen over a diffuse, yellow/reddish background (Figure 1D). Polarised dermoscopy can reveal Biett's sign in dark-phototypes skin, where the vascular structures are less visible. In addition, dermoscopy can be very useful in differentiating the secondary syphilis lesions from other dermatological conditions exhibiting scaling collarettes, including pityriasis rosea, erythema multiforme, actinic porokeratosis, tinea corporis, erythema annulare centrifugum, granuloma annulare, annular variants of psoriasis, and subacute or discoid lupus erythematosus.

\section{Resolution/worsening}

Resolution of untreated manifestations of secondary syphilis can typically take from 4 to 12 weeks. High-risk factors (HIV infection with low CD4 count, malnutrition, MSM, previous syphilis, diabetes, and alcohol abuse) may induce lues maligna, or noduloulcerative syphilis, with asymmetric ulcers or round necrotic plaques with heaped up lamellar or rupioid crusts on the scalp, face, trunk, and extremities.

\section{Latent Syphilis}

Latent syphilis follows the untreated secondary stage, without clinical manifestations. In this phase, the infection can only be detected through serological testing. 16,21,22 Early latent syphilis can occur between the primary and secondary stages or after the resolution of secondary stage. The CDC defined a 1-year cut-off in order to differentiate between early latent and late latent syphilis; it has been highlighted that highest rate of recurrences (about 25\% of treated patients) happen in the first year after infection. 16,21,22 In any stage of syphilis, it is possible to have an asymptomatic or symptomatic neurologic involvement with abnormal cerebrospinal fluid findings. ${ }^{14,16}$

\section{Tertiary Syphilis}

Tertiary, or late syphilis, is a systemic, multiorgan evaluation that occurs in approximately one- third of untreated but infected patients, ${ }^{23,24}$ after a period of years or even decades, including late neurosyphilis (general paresis or tabes dorsalis), cardiovascular syphilis, or gummatous syphilis. Cutaneous lesions (noduloulcerative or gummatous) occur in $16 \%$ of patients with late clinical manifestations; these are usually single, unilateral, and asymptomatic. ${ }^{23,24}$ Noduloulcerative lesions are superficial brown nodules, with serpiginous ulcerative evolution and central resolution. On the other side, gummas are painless destructive rubbery nodules that evolve into punched out ulcers that can grow to many centimetres and that drain necrotic material, invading deeply into tissue and bone, and healing with deeply retracted scars. The neurological involvement in tertiary syphilis is rare in the antibiotic era, but it could be either meningovascular or parenchymatous. ${ }^{14}$ Meningovascular syphilis usually occurs from 5 to 12 years after contagion and manifestations may include haemiplegia, aphasia, and seizures. Parenchymatous manifestations are differentiated in general paresis (irritability and cognitive and memory impairments, emotional lability, and paranoia) and while tabe dorsalis (ataxia, lightning pains, visceral crises, rectal incontinence, and bladder disturbances). ${ }^{25,26}$ Auricular and ocular syphilis can occur during any stage of infection with tinnitus and/or hearing loss, uveitis, retinitis, and retinal detachment. ${ }^{18}$ Cardiovascular syphilis causes an inflammation of the inner lining of the artery (endarteritis) ${ }^{7}$ and may lead to the development of aortic aneurysms (often involving the ascending aorta), aortic insufficiency, coronary-artery stenosis, and myocarditis. Syphilis in pregnancy carries significant risks for adverse outcomes, with more than $25 \%$ of affected pregnancies ending in stillbirth or spontaneous abortion.,22,23

\section{Congenital Syphilis}

Congenital syphilis occurs if the T. pallidum is transmitted from the mother to the fetus. If the infection is transmitted within the first trimester of pregnancy, the consequences may include premature delivery, spontaneous abortion, stillbirth, nonimmune hydrops, or perinatal death. ${ }^{27}$ If the infection is transmitted during the second to third trimester of pregnancy, the majority of infants born to mothers with untreated syphilis appear healthy and have no clinical or laboratory 
evidence of infection at birth, but may develop manifestations of disease months to years later if left untreated. ${ }^{27}$ Thus, congenital syphilis can be differentiated in early congenital (manifestations $\leq 2$ years of age) and late congenital syphilis (manifestations $\geq 2$ years of age). Signs described in early congenital syphilis include skin exfoliative rash (pemphigus syphiliticus), splenomegaly, adenopathy, condylomata lata, pseudoparalysis of Parrot, chorioretinitis, cataracts, periostitis, osteochondritis, nephrotic syndrome, pancreatitis, myocarditis, gastrointestinal malabsorption, and hypopituitarism (diabetes insipidus). Signs described in latent congenital syphilis include Hutchinson's teeth, Mulberry molars, interstitial keratitis, healed chorioretinitis, intellectual disability, hydrocephalus, seizures, optic nerve atrophy, juvenile general paresis, cranial nerve palsies, frontal bossing, saddle nose deformity, protuberant mandible, short maxilla, high palatal arch, saber shin, sternoclavicular joint thickening (Higouménakis sign), and clutton joints.

\section{LABORATORY TESTS AND DIAGNOSIS}

Dermatologists most often diagnose primary or secondary syphilis by observing mucocutaneous manifestations, ${ }^{17-20}$ but every clinical hypothesis must be supported by laboratory confirmation..$^{28-37}$ Although Syphilis was first recognised in Europe in the late 15th century, interpretation of serological tests, diagnosis, and management are often challenging. ${ }^{28-30}$ Syphilis can be diagnosed directly by the tissue, or indirectly by means of serologic tests. To date, multiple assays have been used for laboratory confirmation, with variable ranges of diagnostic accuracy, as summarised in Table 1. ${ }^{30-41}$

\section{Direct Diagnosis}

\section{Microscopic observation}

Since T. pallidum cannot be grown in culture, the direct diagnosis is obtained by the detection with darkfield microscopy of the spirochaetes in fluid or smears procured from a lesion. Indeed, the $T$. pallidum is a corkscrew, spiral-shaped organism that is 6-15 $\mu \mathrm{m}$ long and 0.1-0.2 $\mu \mathrm{m}$ wide, thus it cannot be visualised by direct microscopy and requires darkfield microscopy. This approach has high sensitivity and specificity but requires a specific microscope (paraboloid) and adequately trained physicians, so it is limited to specialised centres. ${ }^{14}$ The CDC initially approved darkfield microscopy and PCR as criteria for the direct detection of $T$. pallidum in tissue specimens. ${ }^{37}$ T. pallidum can also be visualised using immunofluorescent staining and special silver stains, nowadays limited to few centres.

\section{Histopathology}

The histopathological features reported for the primary syphilis lesion are nonspecific and limited by the ulcerative nature of the lesion itself, including ulceration, exocytosis, dense perivascular infiltrate of lymphocytes and plasma cells, and endothelial cell proliferation. ${ }^{28} \mathrm{~A}$ bioptic specimen is more commonly obtained from papular/macular lesions of secondary syphilis. ${ }^{29}$ The range of histopathologic findings that can be exhibited is wide, and include neutrophils in the stratum corneum, irregular/psoriasiform acanthosis, effacement of the rete ridges/ elongated rete ridges, vacuolar interface with vacuolar predominance/with equal numbers of lymphocytes and vacuoles, endothelial swelling, presence of plasma cells, lymphocytes with ample cytoplasm, and interstitial inflammation. ${ }^{29}$ Globally, the main histologic patterns reported are the combined pattern of lichenoid and perivascular inflammatory infiltrate (Figure 2A and B). ${ }^{30}$ Unfortunately, these histopathologic features can be seen in those conditions clinically mimicking secondary syphilis popular/macular lesions, as seen with pityriasis rosea, pityriasis lichenoides, or early mycosis fungoides cases. ${ }^{28}$ Thus, the histopathologic examination has low specificity and low sensitivity and should always be performed along with serology. ${ }^{28-39}$ Moreover, spirochaetes can be identified by silver-based stains, the marking includes melanin too, causing overlap and difficulty in interpreting images (Figure 2C).

\section{Immunohistochemistry}

Immunohistochemistry using a monoclonal antibody to $T$. pallidum can be performed on bioptic specimens. ${ }^{30,31}$ This method is particularly useful to reach the correct diagnosis in retrospective investigations, especially when the diagnostic suspect of syphilis was not considered on a clinical ground. The immunohistochemistry assays developed to date are multiple, and more 
recently were focussed on the recombinant protein technology. Briefly, the T. pallidum DNA derived from Nichols strain genome were amplified by PCR and inserted into an expression vector and then to Escherichia coli cells for expression of fusion proteins with a tag sequence for efficient chromatography purification. The obtained recombinant proteins were tested as antigens in either enzyme-linked immunosorbent assay (ELISA) (e.g., Alere Determine ${ }^{\text {TM }}$ Syphilis TP test; Abbott, Chicago, Illinois, USA) or Western blot format. The ELISA assay appeared to be very sensitive in the detection of T. pallidum, with reported rates of detection of the spirochaetes in both the epidermis and dermis, or in the dermis or epidermis only of $52 \%, 24 \%$, and $20 \%$, respectively. ${ }^{30}$

\section{Indirect Diagnosis}

The indirect diagnosis of syphilis currently relies on serological tests for the detection of antibodies. Two types of serologic tests for syphilis, nontreponemal and treponemaspecific tests (i.e., assays for detection of specific antibodies), were largely validated for diagnostic confirmation of syphilitic infection. ${ }^{32-38}$ However, they are affected by low specificity in some conditions (Table 1). ${ }^{30-41}$ Thus, the research in the last year was focussed on the immunoproteasome of the T. pallidum, aimed to discover new highly-specific antigens and select the optimal antigen combinations for an ideal diagnostic ' $T$. pallidum panel'.39 Among them, surface-exposed proteins, adhesins, and periplasmic and flagellar proteins appeared the most promising. More recently, biochips (i.e., microarrays with orderly located molecular probes for realisation of an appreciable number of specific recognition reactions with the minimum volumes of analysed biological mater) were studied to expand the panel of $T$. pallidum antigens and recombinant proteins to be included in a unique serologic assay. A recently proposed 'immunochip' reached high diagnostic accuracy by adding new synthetic proteins (Tp0277, Tp0319, Tp0453, Tp0684, Tp0965, and Tp1038) to the already known panel of immunodominant antigens (Tp15, Tp17, Tp47, and TmpA). ${ }^{33}$ These methods are now proposed for serological screening, allowing multiple parallel detection of specific serum IgG. 32,33

\section{Nontreponemal tests}

Nontreponemal tests include rapid plasma regain (RPR) and venereal disease research laboratory (VDRL) tests. Both measure tissue damage caused by syphilis by detecting antibodies to cardiolipin, cholesterol, and lecithin, which are normal components of human cells., ${ }^{9,21,22}$ When a nontreponemal test is reactive, the laboratory quantifies the amount of antibody present, expressing it as a titer. Monitoring titers over time enables the assessment of response to treatment. In many patients who are successfully treated for syphilis, a nontreponemal test ultimately becomes nonreactive (in approximately 3 months). ${ }^{34-40}$ Persistently reactive nontreponemal tests after successful treatment typically with low titers (1:1 to $1: 4$ ) and more commonly in patients infected with HIV are called serofast reactions. ${ }^{42}$ False positive and false negative nontreponemal test results can occur in numerous conditions. ${ }^{43}$ False positive results can occur due to patient-related factors (i.e., intravenous drug usage, pregnancy), some autoimmune and inflammatory diseases (i.e., systemic lupus erythematosus, arthritis, ulcerative colitis, thyroiditis, vasculitis), a plethora of infectious diseases (i.e., lymphogranuloma venereum, hepatitis C virus, Epstein-Barr virus, endocarditis, leprosy, varicella, measles, mumps, malaria pinta, yaws, rickettsia, brucellosis, chancroid, tuberculosis, Lyme disease), and even in case of malignancy (e.g., colon carcinoma, lymphomas, metastatic solid cancer). Conversely, false negative results can occur in case of HIV infection, in pregnancy, or in neurosyphilis/lues maligna.

\section{Treponemal tests}

Treponemal tests measure IgM and IgG specific antibodies against $T$. pallidum using native or sonically disrupted T. pallidum cells as the source of total number of antigens. Some of the first developed treponemal tests include the T. pallidum particle agglutination (TPPA), the fluorescent treponemal antibody absorption, and the T. pallidum haemagglutination (TPHA) tests. More recent treponemal assays include enzyme immunoassay and chemiluminescence immunoassay. Treponemal tests are typically more sensitive than nontreponemal tests during early infection. 

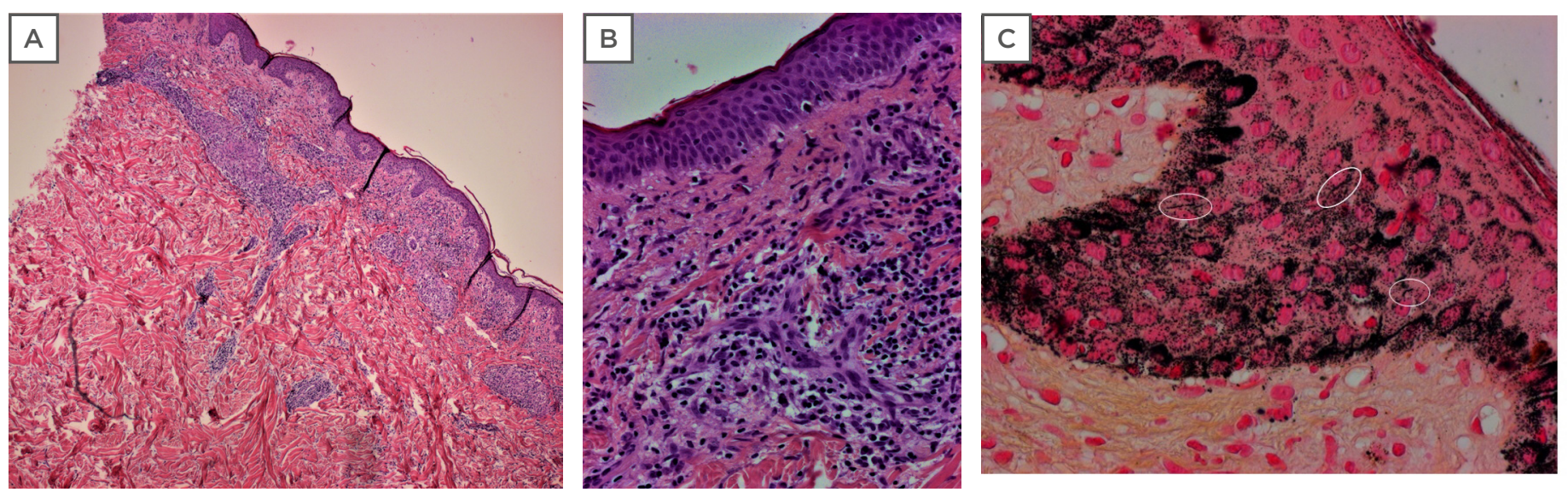

Figure 2: Histopathologic aspects of secondary syphilis.

A) Lichenoid pattern of secondar syphilis characterised by a superficial band-like infiltrate with focal infiltration of the epidermis and a deep perivascular dermal infiltrate (haematoxylin and eosin, original 400x magnification); and B) dermal infiltrate of plasma cells and histiocytes with swollen endothelium; C) Warthin-Starry silver nitrate-based stain highlights both spirochaetes (white circles) and melanin granules resulting in marked background artifacts (haematoxylin and eosin, original 400x magnification).

Table 1: Comparison of the accuracy of the different diagnostic tests used to date in primary syphilis. ${ }^{30-41}$

\begin{tabular}{|l|l|l|}
\hline & Sensitivity (\%) & Specificity (\%) \\
\hline DF microscopy & $62.0-92.0$ & N/A \\
\hline T. pallidum PCR & $72.3-77.8$ & $93.1-100.0$ \\
\hline VDRL & $62.5-78.4$ & $96.0-99.0$ \\
\hline TPHA & $95.0-100.0$ & 77.5 \\
\hline TPPA & $86.2-100.0$ & $99.6-100.0$ \\
\hline RPR & $62.5-76.1,77.0-99.0$ & $93.0-99.0$ \\
\hline FTA-ABS & $72.0-100.0$ & $87.0-100.0$ \\
\hline ELISA & $96.0-100.0$ & $90.0-98.0$ \\
\hline Immunochip & 94.1 & 100.0 \\
\hline
\end{tabular}

DF: darkfield microscopy; ELISA: enzyme-linked immunosorbent assay based on recombinant Treponema pallidum antigen; FTA-ABS: fluorescent treponemal antibody absorbed test; N/A: not assessed; RPR: rapid plasma regain; $T$. pallidum: Treponema pallidum; TPHA: Treponema pallidum haemagglutination assay; TPPA: Treponema pallidum particle agglutination assay; VDRL: venereal disease research laboratory test.

$\lg M$ and $\lg G$ are often detectable 2 and 4 weeks after exposure, respectively, and in some cases within 3 days of a chancre's appearance. Regardless of stage or nonserologic tests, all patients with syphilis should undergo serologic testing with both nontreponemal and treponemal (if not known to have reactive treponemal tests because of a history of syphilis) tests. Treponemal tests are always sequent to nontreponemal tests in order to confirm or to exclude diagnostic hypothesis. ${ }^{34-42,44,45}$

\section{Cerebrospinal fluid examination}

Referrals for cerebrospinal fluid examination via lumbar puncture are recommended only for patients who meet one of three conditions: 1) 
signs or symptoms of neurosyphilis, otic syphilis, or ocular syphilis; 2) suspected treatment failure; or 3) tertiary syphilis. ${ }^{44,45}$ Patients with syphilis who have ocular symptoms should be referred for an ophthalmologic evaluation in addition to cerebrospinal fluid examination.

\section{Test Interpretation}

Interpretation of either can be complicated, particularly when treponemal and nontreponemal tests are discordant. ${ }^{28-35}$ Dermatologists most often diagnose primary or secondary syphilis, which have mucocutaneous manifestations. For patients with current reactive serologies without signs or symptoms of primary, secondary, or tertiary syphilis, physicians must ascertain whether the serologies represent previously treated infection (and if so, whether treatment was successful) or new infection (and if so, determine duration of infection to stage the patient). Doing so requires eliciting a sexual and medical history and reviewing the patient's serologic history, if available.

\section{Congenital syphilis}

Maternal syphilis screening is part of routine standard of care in all pregnancies in many countries, including VDRL/RPR and TPHA/ TPPA. Histological examination of the placenta and/or of the cord may reveal spirochaetes or exhibit signs of enlarged hypercellular villi, proliferative vascular changes, and acute and/or chronic villi. Adequate treatment with penicillin during pregnancy is $98 \%$ effective at preventing congenital syphilis. If the infection is left undiagnosed, treponemal infection is transferred, but most neonates are completely asymptomatic at birth. However, by the age of 3 months, newborns develop hepatomegaly, jaundice, rhinitis, generalised lymphadenopathy, or rash; serologic tests are thus required (VDRL/ RPR and FTA-ABS or TPHA/TPPA). After 2 years of age, gummas and perioral fissures/ scarring; facial changes, including frontal bossing, saddle nose, and prominent maxilla; anterior bowing of the shin; Hutchinson's teeth; intellectual disability; cranial nerve palsies; sensorineural hearing loss; and changes in vision (e.g., interstitial keratitis, secondary glaucoma, and corneal scarring) can occur. At this stage, long bone radiographs, chest $X$-ray, and head X-rays can be performed. Long bone radiographs may show pathologic fractures, metaphyseal serration, localised demineralisation, and osseous destruction, chest X-ray may show diffuse opacification of both lung fields. ${ }^{30}$

\section{TREATMENT}

\section{Pre-penicillin Era}

There were multiple syphilis treatments in the pre-penicillin era, and included, across the centuries, purgative agents, heat, and pyrogens. In the 19th century, mercury was widely used as oral compounds or prepared in topical salves, injections, and even fumigation, but treatment could last for years before being effective. ${ }^{43}$ In 1908, Paul Ehrlich was awarded the Nobel Prize for the discover of arsphenamine (i.e., Salvarsan), an arsenical compound that can be considered the first modern antimicrobial agent. It was introduced at the beginning of the 1910s as the first effective treatment for syphilis and African trypanosomiasis. Salvarsan came to be used in combination with bismuth or mercury, and over 30 doses were recommended to prevent relapse and were supposed to be effective to reduce the risk of neurosyphilis, but this progression occurred in many patients anyway. Then in 1927, a Nobel prize was awarded to Julius WagnerJauregg for the invention of malariotherapy to treat neurosyphilis, which was based on the rational that inoculating patients with Plasmodium vivax induced a fever that was able to kill heat-sensitive $T$. pallidum bacteria. ${ }^{46}$ At the time, Wagner-Jauregg concluded that malariotherapy was more effective the earlier it was used in the course of the disease and recommended combined treatment with Salvarsan. However, from 1925 to 1938, a considerable number of patients were unable to sustain this therapy, with different reported rates of mortality from $3 \%$ to $30 \%$.

\section{General Indications}

Since 1943, penicillin has been an effective treatment for syphilis; with no reported resistance cases to date, it remains the recommended therapy. Benzathine penicillin G is the recommended treatment for syphilis in all stages. ${ }^{36-41}$ However, it is important to estimate the schedule of treatment according to the stage, eventual pregnancy status, a possible allergy, 
or whether the patient has neurosyphilis, otic syphilis, or ocular syphilis. In any case of allergy, it is suggested to use doxycycline $100 \mathrm{mg}$ twice a day for 14 days in early syphilis and for 28 days in the late stage. Physicians should consider empiric treatment if, based on clinical presentation and epidemiologic risk, the index of suspicion is high, particularly if follow-up cannot be assured. Of note, CDC treatment recommendations for all stages and for neurosyphilis, otic syphilis, and ocular syphilis do not depend on HIV status or nontreponemal test titer. ${ }^{44}$ The therapeutic schedule must be extended to the patient's partners in the same way. Treatment response is assessed clinically and serologically. ${ }^{44,45}$

\section{Primary, Secondary, and Early Latent Syphilis}

Treatment for both primary, secondary, and early latent syphilis consist of one administration of benzathine penicillin G 2.4 million units intramuscularly.

\section{Late Latent Syphilis}

The treatment consists of benzathine penicillin $G$ 2.4 million units intramuscularly once a week for 3 consecutive weeks.

\section{Tertiary Syphilis}

Neurosyphilis, otic syphilis, and ocular syphilis require treatment with intravenous aqueous crystalline penicillin $\mathrm{G}$ for $10-14$ days.

\section{Neonatal Syphilis}

As benzathine penicillin $G$ is the only therapy with documented efficacy for treating both the pregnant individual and the fetus, pregnant patients must be desensitised and treated with penicillin. ${ }^{44,45}$

\section{Treatment Reactions}

Patient should be adequately and completely warned about treatment reactions. For example, penicillin $\mathrm{G}$ treatment might precipitate a JarischHerxheimer reaction, which typically presents within 1 day as fever, headache, myalgia, and possibly worsening rash. ${ }^{47-49}$ The hypothesised mechanism underlining the reaction could be related to a massive destruction of spirochaete, causing the release of lipoproteins, immune complex formation, and cytokine cascades, including TNFa, IL-6, and IL-8. ${ }^{47}$ However, it is known that the reaction occurs more commonly and severely in patients with a higher clinical burden of disease and higher nontreponemal test titers. Often mistaken for a drug allergy, the reaction resolves spontaneously, typically within 24 hours. Antipyretics and hydration can be used for symptomatic relief. Pretreatments, such as acetaminophen and antihistamines, do not prevent the reaction.

\section{MANAGEMENT AND FOLLOW-UP}

\section{Response to Treatment}

Clinical and serologic follow-up should follow a determined interval at 1, 3, and 6 months after therapy. In addition, even if a nontreponemal test has been performed recently, a repeat nontreponemal test on the day of treatment should be performed to enable the evaluation of serologic response to treatment. ${ }^{44,45}$

\section{Follow-up of HIV-uninfected Population}

The CDC recommends clinical evaluation and nontreponemal tests at 6, 12, and 24 months for patients without HIV infection and at 3, 6, 9, 12, and 24 months for patients with HIV infection..$^{50}$ Serologic response is defined as a 4-fold or greater decline in nontreponemal test titers (e.g., from 1:64 to 1:16 or lower). Treatment failure should be considered if titer decline does not occur by 1 year for patients without HIV infection with primary or secondary syphilis and by 2 years for patients with HIV infection with early nonprimary, nonsecondary syphilis.

\section{Follow-up of HIV-infected Population}

Treatment failure should be considered if titer decline does not occur by 2 years for patients with HIV infection with primary, secondary, or early nonprimary, nonsecondary syphilis. ${ }^{51}$

\section{Treatment Failure}

Patients should be counselled to return for care if symptoms fail to resolve within 2 weeks. Suspected treatment failure warrants additional evaluation and management that is beyond the scope of this article. Lack of titer decline (called serologic nonresponse) affects $12-20 \%$ of patients with primary and secondary syphilis and is associated with lower baseline nontreponemal 
titer, older age, late-stage infection, and possibly HIV infection. In addition, rising titers in a recently treated patient might indicate reinfection, which is not uncommon among patients with a history of syphilis. ${ }^{41,52}$

\section{Additional Sexually Transmitted Infection Testing and HIV Prevention}

Patients who are diagnosed with syphilis should be collaterally tested for HIV, hepatitis, and other STD, with particular attention to sexual-related vaccinations and pre-exposure or postexposure prophylaxis for HIV. The CDC recommends HIV testing for all patients with syphilis who are not known to have HIV.42,44,45 In addition, a syphilis diagnosis within the previous 6 months is one CDC criterion for initiating pre-exposure prophylaxis. Among males (including MSM), a syphilis diagnosis is associated with a higher risk for subsequent HIV seroconversion. Eliciting a sexual and gender identity history, including the gender(s) of sexual partners, is essential to assess patients' sexual health needs. Physicians should encourage patients with syphilis to disclose their diagnosis to recent sex partner(s) to improve those persons' health and to stop onward transmission. Health departments can also assist with partner notification, if patients desire.

\section{Ethical Considerations}

Syphilis is a legally reportable disease for clinicians. Mechanisms and details of reporting depend on state or local regulations. Physician reporting, which includes stage, enables health departments to prioritise outreach efforts to primary and secondary syphilis patients who are likely to have been infectious to recent sex partners and helps with disease surveillance. Informing patients that the case will be reported, and that the public health department might follow-up with them, can facilitate subsequent positive interactions. Infants and children who are diagnosed with syphilis should have birth and maternal records reviewed to assess whether the infection was congenitally or sexually acquired. Management should be co-ordinated with a paediatric infectious disease specialist and should include evaluation for sexual abuse, typically by consulting child protection services. ${ }^{44,45}$

\section{CONCLUSIONS}

Syphilis, which appeared to be long forgotten before the 70s, is currently considered a public health problem. Diagnosis is still obtained late in most cases, and proper patient management and follow-up are not performed. Syphilis prevention and control efforts by national, state, and local public health agencies include disease surveillance, epidemiologic analyses, education of providers and the public, support for clinical and prevention services, outreach to recently diagnosed patients and their sex partners, and screening of persons who are at high risk for syphilis.

As summarised in the present review, that illustrates the current state of the art of syphilis diagnosis and treatment, the dermatologist who adequately knows syphilis can play a key role in providing the early and correct diagnosis and setting up in the proper management of patients.

\section{References}

1. Newman L et al. Global estimates of the prevalence and incidence of four curable sexually transmitted infections in 2012 based on systematic review and global reporting. 2015;10(12):e0143304

2. World Health Organization (WHO). Report on global sexually transmitted infection surveillance 2015. 2015. Available at: https:// apps.who.int/iris/bitstream/hand le/10665/249553/9789241565301eng.pdf?sequence $=1$. Last accessed: 8 February 2021

3. Hook EW. Syphilis. Lancet 2017; 389:(10078):1550-7.
4. Public Health England (PHE). Infection report: sexually transmitted infections. 2015. Available at: https://assets.publishing.service. gov.uk/government/uploads/ system/uploads/attachment_data/ file/437433/hpr2215_STI_NCSP_ v6.pdf. Last accessed: 12 January 2021.

5. Forrestel AK et al. Sexually acquired syphilis: historical aspects, microbiology, epidemiology, and clinical manifestations. J Am Acad Dermatol. 2020;82(1):1-14.

6. Melo FL et al. Syphilis at the crossroad of phylogenetics and paleopathology. PLoS Negl Trop Dis. 2010;4(1):e575.

7. Giacani L, Lukehart SA. The endemic treponematoses. Clin Microbiol Rev. 2014;27(1):89-115

8. Darrow WW et al. Risk factors for human immunodeficiency virus (HIV) infection in homosexual men. Am J Public Health. 1987;77(4):479-83.

9. Hook EW III. Syphilis and HIV infection. J Infect Dis. 1989;160(3):530-4.

10. Stamm WE et al. The association between genital ulcer disease and the acquisition of HIV infection 
in homosexual men. JAMA

1988;260(10):1429-33.

11. Ghanem KG et al. The modern epidemic of syphilis. N Engl J Med. 2020;382(9):845-54.

12. Centers for Disease Control and Prevention (CDC). Sexually transmitted disease surveillance 2018. 2018. Available at: https://www.cdc. gov/std/stats18/STDSurveillance2018full-report.pdf. Last accessed: 12 January 2021.

13. Beymer MR et al. Sex on demand: geosocial networking phone apps and risk of sexually transmitted infections among a cross-sectional sample of men who have sex with men in Los Angeles County. Sex Transm Infect. 2014;90(7):567-72.

14. Harmon ED, Robertson EW. Syphilis: a growing concern. Nurse Pract. 2019:44(8):21-8.

15. Centers for Disease Control and Prevention (CDC). Syphilis in pregnancy - 2015 STD Treatment Guidelines. 2015. Available at: https:// www.cdc.gov/std/tg2015/syphilispregnancy.htm. Last accessed: 12 January 2021.

16. Hook EW 3rd, Marra CM. Acquired syphilis in adults. N Engl J Med. 1992;326(16):1060-9.

17. O'Byrne P, MacPherson P. Syphilis BMJ. 2019:365:14159.

18. Katz AR et al. Dermatologically challenging syphilis presentation. Int J STD AIDS. 2019;30(7):707-9.

19. Tognetti L et al. Unusual presentation of secondary syphilis: membranoproliferative glomerulonephritis and mucocutaneous lesions. Int J STD AIDS. 2018:29(4):410-3.

20. Tognetti $L$ et al. Syphilitic alopecia: uncommon trichoscopic findings. Dermatol Pract Concept. 2017;7(3):55-9

21. Arando Lasagabaster M, Otero Guerra L. Syphilis. Enferm Infecc Microbiol Clin. 2019;37(6):398-404

22. Barnett R. Syphilis. Lancet. 2018;391(10129):1471.

23. Jorge LM et al. Tertiary syphilis: tubero-serpiginous and tuberoulcerous syphilids. Braz J Infect Dis. 2016;20(3):308-9.

24. Tampa $M$ et al. Brief history of syphilis. J Med Life. 2014;7(1):4-10.

25. Olry R, Haines DE. Tabes dorsalis: Not, at all, "Elementary my dear Watson!". J Hist Neurosci. 2018;27(2):198-203.

26. Gonzalez $\mathrm{H}$ et al. Neurosyphilis. Semin Neurol. 2019;39(4):448-55
27. Arrieta AC, Singh J. Congenital Syphilis. N Engl J Med. 2019;381(22):2157.

28. Engelkens $\mathrm{HJ}$ et al. Primary and secondary syphilis: a histopathological study. Int J STD AIDS. 1991;2(4):280-4

29. Flamm A et al. Histopathologic features distinguishing secondary syphilis from its mimickers. J Am Acad Dermatol. 2020;82(1):156-60.

30. Rato $M$ et al. Syphilis: relevance of immunohistochemistry for the diagnosis. JAAD Int. 2018;79(3):AB277.

31. Wang LN et al. [Sensitivity and specificity of ELISA based on recombinant Treponema pallidum antigen and rapid plasma reagin test in diagnosis of syphilis: a comparative study]. Zhonghua Yi Xue Za Zhi. 2007;87(24):1721-2. (In Chinese).

32. Kubanov A et al. Novel Treponema pallidum recombinant antigens for syphilis diagnostics: current status and future prospects. Biomed Res Int. 2017;2017:1436080.

33. Runina AV et al. Immunochip for syphilis serodiagnostics with the use of extended array of Treponema pallidum recombinant antigens. Bull Exp Biol Med. 2018;165(6):767-71.

34. Ortiz DA et al. The traditional or reverse algorithm for diagnosis of syphilis: pros and cons. Clin Infect Dis. 2020;71(1):S43-51.

35. Morshed MG, Singh AE. Recent trends in the serologic diagnosis of syphilis. Clin Vaccine Immunol. 2015;22(2):13747.

36. Park IU et al. Performance of treponemal tests for the diagnosis of syphilis. Clin Infect Dis. 2019;68(6):913-8.

37. Tuddenham S et al. Syphilis laboratory guidelines: performance characteristics of nontreponemal antibody tests. Clin Infect Dis. 2020:71(Suppl 1):S21-42.

38. Park IU et al. Sensitivity and specificity of treponemal-specific tests for the diagnosis of syphilis. Clin Infect Dis. 2020;71:S13-20.

39. Vrbová E et al. A retrospective study on nested PCR detection of syphilis treponemes in clinical samples: PCR detection contributes to the diagnosis of syphilis in patients with seronegative and serodiscrepant results. 2020;15(8):e0237949.

40. Cornelisse VJ et al. Getting to the bottom of it: sexual positioning and stage of syphilis at diagnosis, and implications for syphilis screening. Clin Infect Dis. 2020;71(2):318-22.
41. Lin LR et al. Further evaluation of the characteristics of Treponema pallidum-specific IgM antibody in syphilis serofast reaction patients. Diagn Microbiol Infect Dis. 2011;71(3):201-7.

42. Young $\mathrm{H}$ et al. The architect syphilis assay for antibodies to Treponema pallidum: an automated screening assay with high sensitivity in primary syphilis. Sex Transm Infect. 2009;85(1):19-23.

43. O'Shea JG. 'Two minutes with venus, two years with mercury'--mercury as an antisyphilitic chemotherapeutic agent. J R Soc Med. 1990;83(6):392-5.

44. Workowski KA et al. Sexually transmitted diseases treatment guidelines, 2015. MMWR Morb Mortal Wkly Rep. 2015;64:(RR-03)1-137.

45. World Health Organization (WHO) WHO guidelines for the treatment of Treponema pallidum (syphilis). 2016. Available at: https://apps. who.int/iris/bitstream/hand le/10665/249572/9789241549806-en g.f;jsessionid=DB5FF67A9897ED272 91AEF0100114D75? sequence $=1$. Last accessed: 8 February 2021.

46. Austin SC et al. The history of malariotherapy for neurosyphilis. 374 Modern parallels. JAMA. 1992;268(4):516-9.

47. Pound MW, May DB. Proposed mechanisms and preventative options of Jarisch-Herxheimer reactions. J Clin Pharm Ther. 2005;30(3):291-5.

48. Yang $\mathrm{CJ}$ et al. Jarisch-Herxheimer reaction after penicillin therapy among patients with syphilis in the era of the HIV infection epidemic: incidence and risk factors. Clin Infect Dis. 2010;51:976-9.

49. Aronson IK, Soltani K. The enigma of the pathogenesis of the JarischHerxheimer reaction. $\mathrm{Br} \mathrm{J}$ Vener Dis. 1976;52(5):313-5.

50. Rolfs RT et al. A randomized trial of enhanced therapy for early syphilis in patients with and without human immunodeficiency virus infection. The Syphilis and HIV Study Group. N Engl J Med. 1997;337(5):307-14.

51. Sena AC et al. Response to therapy following retreatment of serofast early syphilis patients with benzathine penicillin. Clin Infect Dis. 2013;56:420-2.

52. Centers for Disease Control and Prevention (CDC). Preexposure prophylaxis for the prevention of HIV infection in the United States - 2017 update. 2017. Available at: https:// www.cdc.gov/hiv/pdf/risk/prep/cdchiv-prep-guidelines-2017.pdf. Last accessed: 14 January 2021. 